\title{
A TECNOLOGIA DE WORKFLOW COMO IMPORTANTE FERRAMENTA DE APOIO PARA A MODELAGEM, GESTÃO E MELHORIA CONTÍNUA DO PROCESSO EM EDUCAÇÃO A DISTÂNCIA
}

\author{
Valeska Pivoto Patta Marcondes \\ Instituto Nacional de Telecomunicações - INATEL \\ valeska@inatel.br \\ Germano de Souza Kienbaum, PhD \\ Instituto Nacional de Pesquisas Espaciais - INPE \\ kienbaum@lac.inpe.br \\ Solon Venâncio de Carvalho, Dr. \\ Instituto Nacional de Pesquisas Espaciais - INPE \\ solon@lac.inpe.br
}

\begin{abstract}
Resumo
Este trabalho faz uma breve revisão do uso da tecnologia de worflow em apoio a ambientes virtuais de aprendizagem em geral e apresenta um caso particular de desenvolvimento e aplicação de um módulo ou ferramenta baseado nesta tecnologia, denominado Workflow Course Manager, que foi projetada para ser integrada ao ambiente virtual de aprendizagem TelEduc, de forma a convertê-lo em um sistema completo do tipo Learning Content Management System. A incorporação da tecnologia de workflow a sistemas EAD visa proporcionar aos educadores poderosos recursos para a modelagem, construção, execução e gestão automáticas de cursos que utilizam a Web como meio de comunicação. As capacidades do uso da abordagem e dos sistemas nela baseados para a difusão e a gestão de conhecimento de natureza didática são analisadas, bem como sua potencialidade para contribuir na criação de um ciclo de melhoria contínua do processo em educação a distância.
\end{abstract}

Palavras-chave: EAD, Tecnologia Educacional, Gestão de Processos, Workflow.

\section{THE WORKFLOW TECHNOLOGY AS AN IMPORTANT SUPPORTING TOOL FOR THE MODELING, MANAGEMENT AND CONTINUOUS IMPROVEMENT OF THE DISTANCE EDUCATION PROCESS}

\begin{abstract}
This work presents a brief survey of the use of the workflow technology to support EAD systems in general and the development and implementation of a particular tool of this kind, named Workflow Course Manager, which was designed to be integrated with the TelEduc EAD system, in order to convert it into a complete Learning Content Management System. The incorporation of the workflow technology in EAD systems aims at providing educators with powerful resources for modeling, building, automatic execution and management of course content on the Web. The strengths and weaknesses related with the use of the approach and of its support tools for diffusion and management of knowledge of a didactic nature are further analyzed, as well as its potential to contribute to the creation of a continuous improvement cycle of the distance V. 6 № 1 , Julho, 2008
\end{abstract}


education process.

Key words: Distance Education, Educational Technology, BPM, Workflow.

\section{1 - Introdução}

Os sistemas para Educação a Distância (EAD) atingem um público cada vez maior e mais diferenciado, distribuído globalmente, formado por pessoas que possuem uma grande variedade de habilidades, educação e objetivos, fazendo com que a gestão do processo de ensino-aprendizagem a distância torne-se um constante e crescente desafio.

Este trabalho tem como objetivo propor uma solução de baixo custo que permita a modelagem, a construção, a execução e a gestão automáticas de atividades educacionais, visando dar suporte a todo o ciclo de vida e proporcionar uma melhoria contínua do processo de ensino/aprendizagem, para aplicação a cursos que utilizam a Web como seu meio de comunicação.

A solução proposta considera o sistema TelEduc como plataforma base e propõe sua transformação, por meio da integração de um módulo ou ferramenta de gerência de workflow, denominado Workflow Course Manager (WfCM), para a criação de um ambiente completo de EAD do tipo Learning Content Management System (LCMS).

A estrutura do trabalho tem a seguinte forma: inicialmente apresenta-se a evolução histórica dos ambientes de apoio a EAD; a seguir identifica-se os problemas com a arquitetura dos ambientes de apoio a EAD tradicionais e apresenta-se uma proposta de solução, baseada na incorporação da tecnologia de workflow a estes sistemas; fundamenta-se a metodologia a ser adotada e o escopo da solução proposta; apresenta-se a arquitetura do ambiente e de um protótipo em implementação; faz-se uma revisão dos resultados obtidos por pesquisas anteriores correlacionadas com este trabalho; apresenta-se o estágio de desenvolvimento atual da presente proposta e as diretrizes para sua continuação; e, por último, apresenta-se um sumário do trabalho com suas principais conclusões.

\section{2 - A Evolução dos Sistemas de Apoio a Educação a Distância}

Muitos sistemas para EAD tradicionais foram projetados durante os anos 80 e 90, tendo sido concebidos originalmente para atuar como Sistemas de Gestão de Aprendizagem (Learning Management Systems - LMS), direcionados principalmente para a oferta da grade de cursos e para o apoio ao gerenciamento do processo administrativo de instituições de ensino (Greenberg, 2002).

Os LMS surgiram com o objetivo de auxiliar o planejamento, a disponibilização e o gerenciamento dos eventos dentro de um ambiente educacional virtual, incluindo desde o controle de ferramentas administrativas até ferramentas de comunicação. $\mathrm{O}$ foco dos LMS é gerenciar os aprendizes, mantendo registrada através de ferramentas administrativas a atuação dos mesmos em todos os tipos de atividades de treinamento.

Em adição à gerência das funções administrativas provida pelos sistemas LMS, os Sistemas de Gestão de Conteúdo de Aprendizagem (Learning Content Management Systems - LCMS) disponibilizam ferramentas adicionais que permitem a construção e gestão do conteúdo educacional (Greenberg, 2002).

Segundo (Bicudo, cap 2), a disseminação dos LMS e dos LCMS vem de algum modo constituir um veículo eficaz na construção do saber. Mas, estas plataformas podem se tornar um pesado fardo financeiro para as empresas e instituições de ensino que optarem por uma plataforma comercial como o WebCT (Webct Inc, 2005), V. 6 № 1 , Julho, 2008 
BlackBoard (Blackboard Inc,2005), entre outras.

Como alternativa existem sistemas LMS e LCMS de código aberto dos quais os mais utilizados são o Moodle (Moodle, 2004), o Claroline (Claroline.Net, 2005), o Atutor (Atutor, 2005) e o TelEduc (Núcleo de Informática Aplicada à Educação, 2005), (Bicudo, cap2).

A maioria destes sistemas, mesmo os comerciais, como WebCT (WebCT, 2005) e Blackboard (Blackboard, 2005), caracterizam-se por dar suporte a tarefas individuais de aprendizado e não levam em consideração o processo de ensino/aprendizagem de uma forma abrangente e integrada.

\section{3 - Identificação do Problema e Proposta de Solução}

Vários sistemas LMS tradicionais para EAD são ainda hoje muito difundidos e bastante utilizados, mas possuem como foco, principalmente, as atividades administrativas e de gerenciamento da grade dos cursos. Eles não dispõem, por exemplo, de funcionalidades próprias para a elaboração do material didático, nem para a execução automática ou gestão dos cursos individuais a serem ministrados na Internet com o seu apoio.

Sistemas de EAD tradicionais não oferecem a possibilidade de se modelar os aspectos dinâmicos ou personalizados de um processo ensino-aprendizagem a distância, ou seja, uma maneira na qual o professor, ao criar um curso, crie um roteiro de atividades, de forma a gerar um caminho a ser percorrido por cada aluno, conforme suas necessidades e habilidades.

As limitações de natureza técnica observadas são de dois tipos distintos: o primeiro refere-se à estruturação do material didático de uma forma geral, com a criação de um roteiro lógico a ser percorrido pelos alunos, na forma da execução de um projeto, cuja edificação final a ser construída é o conhecimento que se deseja transmitir; o segundo deles se refere à natureza estática do próprio conhecimento ou conteúdo educacional disponibilizado.

O primeiro caso é caracterizado pelos sistemas que não disponibilizam ferramentas para a auxiliar a construção do conteúdo didático, deixando inteiramente a cargo do professor a melhor forma para sua estruturação, contrariamente ao que se exige no planejamento e desenvolvimento de qualquer projeto em outras áreas de conhecimento. Isto ocorre com boa parte dos sistemas LMS tradicionais, como o Moodle e o próprio TelEduc.

Nestes casos a elaboração do roteiro geral das atividades de aprendizado requer um grande esforço por parte do professor/orientador, uma vez que ele só pode ser feito de forma manual, ou seja, o professor precisa inserir as atividades necessárias gradualmente, usando as ferramentas de apoio disponíveis no sistema, a partir de um plano geral criado por ele separada e antecipadamente.

O segundo caso fica caracterizado pelos sistemas de aprendizagem que apresentam uma natureza estática dos módulos e páginas de conteúdo usadas na exposição do seu material didático. Estes cursos se caracterizam por serem criados com base em um material didático "genérico", que necessita atender um vasto número de situações de aprendizagem e indivíduos diferentes.

Os fatores citados acima descrevem o problema visto por um ângulo puramente técnico, que pode ser superado com o avanço da tecnologia educacional, resultante dos esforços de pesquisa e desenvolvimento de novos sistemas em andamento, tanto na 
academia como no setor privado, visando a criação de ambientes mais flexíveis de aprendizagem, adaptáveis às características peculiares de cada aprendiz.

Há, entretanto um terceiro fator de grande interesse, também ausente nos ambientes de apoio a EAD pesquisados, que diz respeito ao monitoramento e à gestão do conhecimento disponibilizado, objetivando a avaliação e a melhoria contínua do próprio processo de ensino/aprendizagem. A solução para este problema compreende não só a adição de ferramentas da tecnologia educacional, conforme citadas acima, mas também a aplicação do ambiente resultante em sucessivos estudos de casos reais, priorizando-se a coleta de informações e o execução do curso sob diversos cenários, correspondentes a diferentes público alvo e modelos alternativos de construção do material didático. Esta análise pode beneficiar-se também do uso de simulações como forma de complementação aos estudos de casos reais e o conjunto destas experimentações precisa ser avaliado em busca de uma melhoria contínua do processo educacional.

Tanto as deficiências de ordem técnica quanto aquelas provenientes da questão da gestão do conhecimento e melhoria contínua do processo educacional podem, em princípio, se beneficiar da incorporação da tecnologia de gerenciamento automático de processos de negócios em sistemas educacionais, mas não se têm conhecimento da existência de muitas iniciativas deste tipo, seja na área acadêmica ou no setor privado. Segundo (Helic et al, 2005) estas características têm estado ausentes até mesmo dos principais sistemas de aprendizagem comerciais em utilização.

Com o uso crescente da tecnologia da informação no domínio da educação e o aumento do seu público alvo, que passou a estar distribuído globalmente e a possuir uma grande variedade de habilidades, educação e objetivos, as expectativas dos estudantes e professores com relação aos sistemas de apoio ao processo educacional cresceram também significativamente (Lin et al, 2002) e a modelagem e a gestão de uma forma abrangente e integrada do processo ensino-aprendizagem a distância, que atenda a todos os requisitos mencionados acima, tornou-se um grande desafio.

Este trabalho tem como objetivo demonstrar a utilização da tecnologia de workflow, dentro do contexto da educação à distância, visando melhorar a modelagem, a construção, a execução e gestão automáticas do conteúdo didático de cursos na Web, e proporcionar uma forma de melhoria contínua do processo de ensino/aprendizagem, por meio do desenvolvimento de um módulo ou ferramenta para gestão com base na tecnologia de workflow, denominado Workflow Course Manager (WfCM), que possa ser integrado ao sistema TelEduc, de forma a compor um ambiente de ensino a distância mais completo.

\section{4 - Metodologia e Escopo da Proposta}

De um ponto de vista da tecnologia de processos, em um sistema de EAD o processo de negócio em análise é o processo de ensino/aprendizagem. Este processo é bastante dinâmico, uma vez que os aprendizes diferem em seus estilos de aprendizado, habilidades ou nível de conhecimento.

Segundo (Helic et al, 2005) o processo de aprendizagem deve ser visto como sendo uma instância de uma particular estratégia pedagógica que geralmente consiste de:

- Um conjunto de tarefas de aprendizado relacionadas que são executadas de acordo com as regras pedagógicas da estratégia em questão. 
- Um número de participantes cada qual desempenhando um papel dentro do processo (por exemplo, aluno, professor ou tutor).

- Um número de recursos necessários para que se atinja o objetivo de aprendizagem.

- Um número de ferramentas de sistemas interativos que dão suporte aos participantes em suas tarefas individuais, e também a comunicação e colaboração com outros participantes do processo.

Ainda segundo (Helic et al, 2005), devido à natureza dinâmica de um processo de aprendizagem, pode-se definir um ciclo de vida para o mesmo, contendo as seguintes fases:

- Fase de modelagem, onde um processo de aprendizagem em particular é modelado com todos os seus componentes;

- Fase de aprendizado, onde o processo de aprendizado é executado;

- Fase de observação e melhoria, onde o processo de aprendizagem é monitorado, analisado e consequentemente melhorado para suprir uma situação particular.

Este trabalho adota uma abordagem que integra as duas áreas de conhecimento, gestão de processos e tecnologia educacional, com base nas similaridades existentes entre o ciclo de vida de um processo de negócio e o ciclo de vida do processo de ensino/aprendizagem. Utilizando-se esta abordagem integrada, identifica-se, modela-se, implementa-se os processos de elaboração do conteúdo didático, propõe-se uma arquitetura para sua execução (disponibilização) e gestão automáticas, e uma diretriz para a melhoria contínua dos processos envolvidos.

O escopo do estudo e do desenvolvimento desta proposta inclui ainda o projeto e a implementação de um protótipo da ferramenta de workflow para demonstrar suas funcionalidades e integração com o sistema TelEduc, bem como a análise da abordagem proposta num contexto de difusão e gestão de conhecimento de conteúdo didático.

\section{5 - Arquitetura do Ambiente Workflow Course Manager (WfCM)}

O sistema Workflow Course Manager está sendo desenvolvido utilizando uma arquitetura cliente-servidor, que é a arquitetura geral empregada para a elaboração e disponibilização de serviços em Intratnets e na Internet, conforme ilustrada na Figura 1. Desta forma, a partir de qualquer web browser é possível acessar as funcionalidades providas pelo TelEduc e pelo sistema de gerência de workflow integrado.

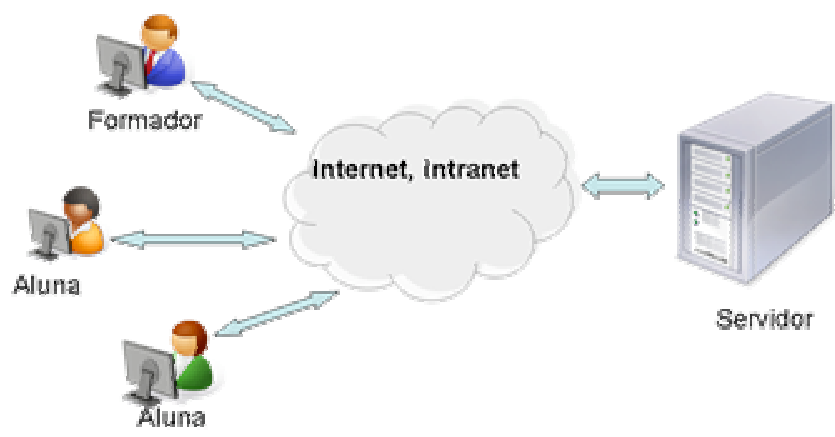

Figura 1 - Arquitetura do Workflow Course Manager (WfCM) 
No lado servidor ficam hospedadas a máquina de workflow, a base de dados que armazena todas as informações persistentes do sistema e o servidor web contendo a aplicação WfCM e o TelEduc. O diagrama da Figura 2 representa os casos de uso do sistema, de acordo com o que se segue:

Os atores Aluno e Formador representam papéis já existentes no sistema TelEduc e que são utilizados pelo sistema WfCM. O Aluno é uma pessoa que interage com o sistema WfCM guiado de acordo com a execução de um workflow previamente criado pelo Formador. O Formador é uma pessoa que interage com o sistema WfCM para criar ou gerenciar um workflow.

O caso de uso Criar Workflow representa o núcleo central do sistema, onde estão definidas as atividades e as regras de negócio que guiam sua execução automática. Ele é responsável por implementar as funcionalidades que permitem a elaboração de um Workflow e demais funcionalidades correspondentes. A elaboração de um Workflow é realizada de forma simples, usando recursos gráficos, permitindo uma fácil operação pelo usuário Formador.

O usuário Formador introduz uma atividade inicial e todas as demais atividades, bem como a conexão entre elas. Ele especifica todas as regras que guiam a execução automática das mesmas, que pode estar baseada em condições verificadas em tempo real, como por exemplo, uma nota de uma prova realizada ao finalizar uma atividade de avaliação.

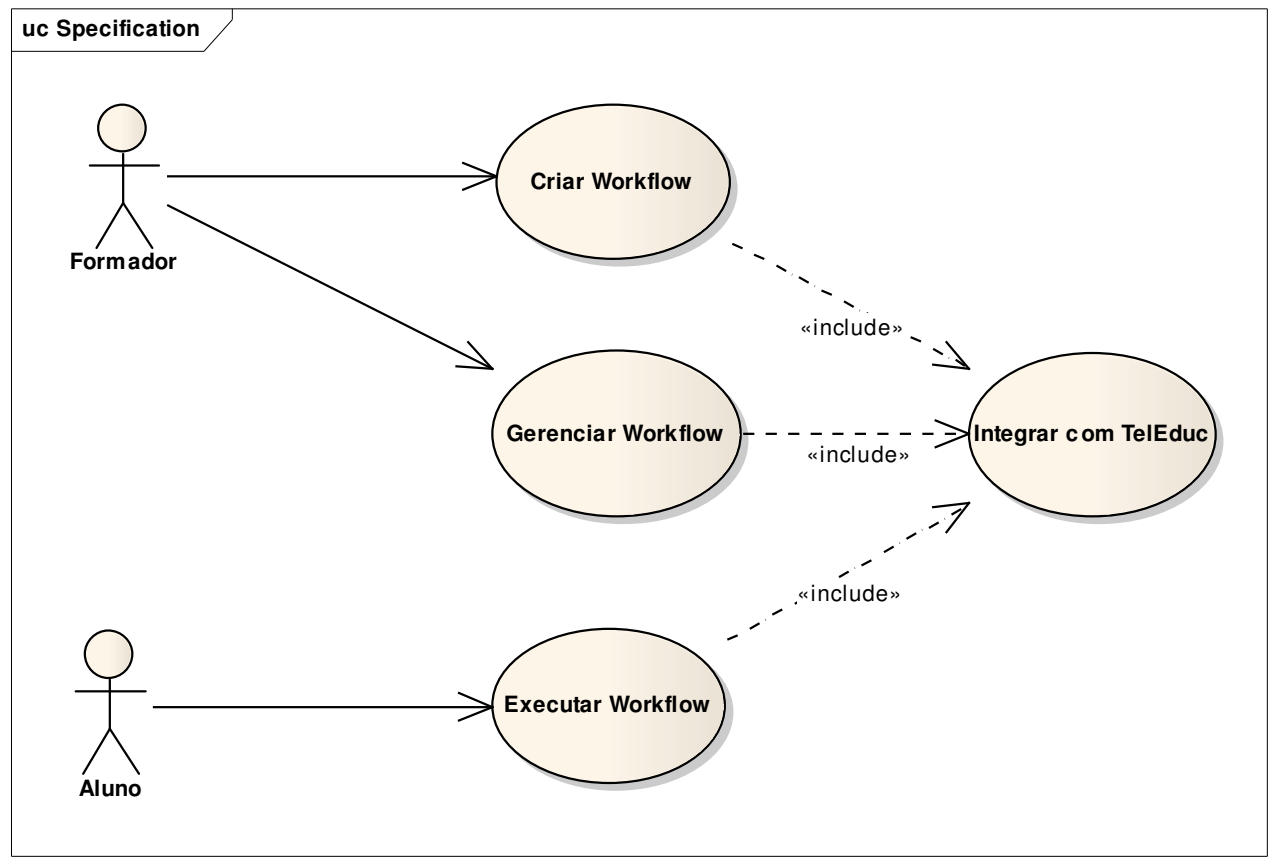

Figura 2 - Diagrama de Casos de Uso do Sistema WfCM

Para cada atividade que compõe o Workflow faz-se uma associação da mesma a um arquivo correspondente a um conteúdo educacional. Ou seja, um arquivo de texto, uma imagem, um vídeo, que é o objeto de estudo. Além disto, o sistema permite a criação de questões objetivas que podem ser associadas a uma atividade de avaliação.

O caso de uso Executar Workflow é responsável por implementar as V. 6 № 1, Julho, 2008 
funcionalidades que permitem ao usuário Aluno executar um curso previamente criado por um Formador. O aluno interage com os conteúdos (mídia) que foram associados a cada atividade do modelo e tem à sua disposição links ou botões que permitem que o roteiro seja percorrido até a sua finalização.

O sistema WfCM mantém as informações persistentes em banco de dados, de forma a não perdê-las quando o usuário sai da aplicação.

O caso de uso Gerenciar Workflow é responsável por implementar as funcionalidades que permitem a recuperação de informações relacionadas aos modelos e sua execução, como por exemplo, relatórios que indicam quais usuários realizaram determinado curso, em que etapa (atividade) cada usuário se encontra no momento, e outras informações relevantes que podem ser adicionadas e apresentadas na forma de gráficos.

O caso de uso Integrar com TelEduc é responsável por implementar a integração das funcionalidades do sistema WfCM com o sistema TelEduc.

\section{6 - Histórico da Pesquisa}

Esta pesquisa está sendo realizada como parte das atividades de pesquisa do Núcleo de Estudos em Modelagem e Simulação de Sistemas (NEMESIS), que tem como um de seus focos principais o desenvolvimento e a aplicação de uma metodologia integrada para modelagem, simulação e gestão automatizada de processos em geral, e sua aplicação em gerência de projetos e educação à distância.

Dentro do contexto da educação à distância, um primeiro protótipo de um módulo para modelagem e gestão do conteúdo didático de cursos a serem disponibilizados com o TelEduc foi implementado em (Bicudo, 2006). As principais funcionalidades disponíveis no protótipo são, a existência de uma interface gráfica amigável e integrada diretamente com o LMS (Learning Management Systems) para a modelagem de cursos, proporcionada pelo Simprocess (CACI, 2004); a disponibilização de ferramentas administrativas para comunicação e colaboração e de ferramentas para avaliação da aprendizagem, disponíveis a partir do TelEduc.

Em (Marcondes et al, 2007) foram apresentados os detalhes de implementação deste primeiro protótipo, suas limitações e um novo protótipo de um módulo para modelagem e gestão do processo educacional, utilizando uma ferramenta de código livre denominado OS Workflow.

Durante o desenvolvimento do segundo protótipo apresentado em (Marcondes et al, 2007) foi observada uma limitação com relação à interface gráfica para modelagem do processo, uma vez que foi necessária a utilização do sistema de edição gráfica Workflow Designer, ou seja, um módulo não integrado à máquina de workflow responsável pela execução do processo.

Com base nas lições aprendidas nos desenvolvimentos anteriores, foi elaborada esta nova proposta, visando identificar uma máquina de workflow que permitisse o desenvolvimento de uma interface gráfica mais adequada. A máquina de workflow selecionada foi o componente Workflow da biblioteca EzComponent (EzComponent, 2008).

\section{7 - Estágio Atual e Diretrizes Futuras}

A biblioteca EzComponent (EzComponent, 2008) fornece vários componentes de propósito geral para desenvolvimento independente ou integrado a uma aplicação PHP. É uma biblioteca livre e de código aberto. Como o sistema TelEduc foi 
desenvolvido na linguagem PHP a integração com o mesmo fica bastante facilitada.

O componente principal a ser utilizado é o Workflow, que fornece as funcionalidades principais de um sistema de workflow baseado em atividades, incluindo a definição e execução da especificação de um workflow.

Outra facilidade fornecida pela biblioteca EzComponents é o componente denominado Workflow Database Tiein que permite a interação com uma base de dados, o que é relevante ao propósito do trabalho.

O sistema WfCM está em fase de desenvolvimento e os resultados obtidos até agora têm atendido as expectativas no que se refere ao cumprimento das especificações do sistema.

A Figura 3 ilustra com um estudo de caso simples alguns elementos envolvidos na criação de um curso.

O modelo representado na Figura 3 corresponde a um curso fictício bem simplificado criado por um Formador, contendo uma seqüência de atividades representadas pelos retângulos e setas.

A cada atividade é atribuída um conteúdo (um arquivo .doc, html, uma imagem, um vídeo, etc.) que estão representados por $\mathbb{W}$

A cada atividade de Avaliação é criada (via TelEduc) uma página que conterá as perguntas e respostas (representada na Figura 3 por $\$$ ) a ser atribuída a esta atividade.

As setas representam o roteiro a ser executado conforme as regras de negócio inseridas.

No diagrama da Figura 3 as setas sólidas e de ponto representam uma decisão que será tomada pelo sistema WfCM para direcionar o usuário Aluno, no momento de execução, a realizar a atividade "Avaliação Y" ou "Conceitos W". Esta decisão será baseada em uma condição (regra) que foi configurada no momento de criação do modelo, e que pode estar associada a uma nota obtida ao final da atividade "Avaliação W". O mesmo acontece para a outra atividade de Avaliação: "Avaliação Y".

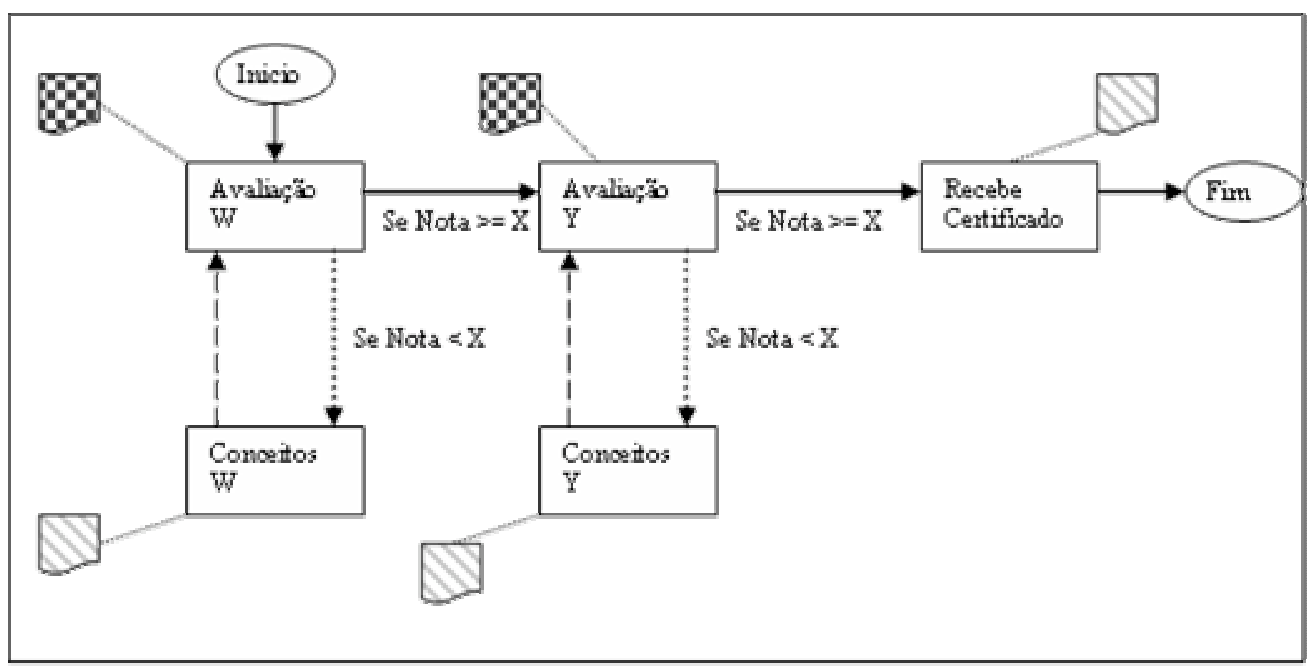

Figura. 3 - Elementos de modelagem de um Curso

A seta em tracejado longo representa uma ação incondicional, ou seja, necessariamente o usuário Aluno será direcionado para a atividade que ela está apontando. Ou seja, após a conclusão da atividade "Conceitos W" o usuário será 
direcionado a realizar a atividade "Avaliação W".

A integração do módulo de gerenciamento de workflow WfCM com o TelEduc deverá ser feita com o menor acoplamento possível, facilitando com isso a instalação, utilização e a manutenção do sistema.

A utilização de ferramentas de código livre permitirá a completa exploração e até mesmo a expansão das funcionalidades disponíveis neste tipo de aplicativo.

Como benefícios diretos do sistema proposto são esperados o monitoramento em tempo real dos cursos, a análise estatística dos dados coletados durante a realização dos mesmos e o aperfeiçoamento do processo ensino-aprendizagem a distância por meio de uma melhor gestão.

\section{8 - Conclusões}

O TelEduc é um ambiente para EAD bastante utilizado na comunidade latina e está baseado numa arquitetura modular, formada pela agregação de diversas ferramentas para o trabalho colaborativo na Internet, típica de sistemas para EAD concebidos para atuar como Sistemas de Gestão de Aprendizagem (Learning Management Systems LMS).

Os sistemas para EAD tradicionais como o TelEduc não dispõem de certas funcionalidades extras capazes de auxiliar nos procedimentos de elaboração de cursos e monitoramento do processo de ensino/aprendizagem, presentes apenas numa nova geração de sistemas EAD conhecidos como Sistemas para Gestão do Conteúdo de Aprendizagem.

Funcionalidades deste tipo geralmente aparecem associadas na literatura ao desenvolvimento de sistemas e serviços relacionados com a gerência de processos (BPM - Business Process Management), sendo sua utilização na área de tecnologia educacional ainda bastante limitada.

Este trabalho descreve uma metodologia para a integração de técnicas da área de gestão de processos com a tecnologia educacional e sua aplicação para o desenvolvimento de um módulo de gerência de processos destinado a ser integrado com ambiente EAD TelEduc, visando transformá-lo em um ambiente mais completo do tipo LCMS. A viabilidade da utilização desta metodologia ficou demonstrada em trabalhos anteriores, como (Kienbaum et al, 2006), pela utilização do ambiente TelEduc e do sistema de simulação Simprocess (CACI, 2004) como plataformas para implementação do ambiente EAD com as características desejadas.

A continuação da pesquisa anterior de acordo com as diretrizes deste trabalho visou a substituição do módulo para modelagem e gestão do conteúdo criado com o uso do aplicativo Simprocess por um protótipo baseado em Workflow de código livre, baseado na biblioteca de classes EZComponent (EzComponent, 2008). A troca da máquina de workflow do Simprocess por uma de código livre possibilitou a completa exploração e a expansão das funcionalidades disponíveis no protótipo anterior, superando algumas dificuldades enfrentadas na primeira implementação.

Como benefícios adicionais da continuidade do desenvolvimento e aplicação do sistema com este novo módulo de modelagem e gestão do conteúdo apresentado são esperados: o monitoramento em tempo real dos cursos; a análise estatística dos dados coletados durante a realização dos mesmos; e o aperfeiçoamento do processo ensinoaprendizagem a distância através de uma melhor gestão do conteúdo educacional do ponto de vista do educador. 
Além de requerer domínio do meio ou sistema de transmissão da informação adotado, ensinar a distância é muito diferente de ensinar presencialmente, solicitando dos formadores diferentes habilidades de apresentação da informação, de planejamento, desenvolvimento e avaliação de estratégias de ensino.

As funcionalidades adicionais do ambiente proposto vão além dos benefícios de natureza meramente tecnológica, e podem ser exploradas para se buscar a solução ou a minimização dos problemas de ordem didática relacionados com o uso de sistemas para EAD, abordando-se as estratégias e cenários envolvidos na difusão do conhecimento de conteúdo acadêmico, assim como aspectos relacionados com a gestão e melhoria contínua do processo educacional, segundo análise contida em (Helic et al, 2005).

Como resultado final desta pesquisa, espera-se que a aplicação da metodologia e ferramentas apresentados se constituam numa relevante contribuição para a comunidade de usuários do TelEduc, pela expansão e criação de novas funcionalidades, e manutenção do mesmo como uma solução alternativa de baixo custo para EAD.

Por fim, cabe observar ainda que, independentemente do grau de sofisticação tecnológica que venha a ser adotada, a EAD é apenas uma entre diversas alternativas pedagógicas e ela pode e deve incorporar as novas tecnologias como meio para alcançar os objetivos das práticas educativas que se deseja implementar, mas não pode perder de vista as concepções de homem e sociedade assumidas, bem como a real situação e necessidades das populações a que se pretende servir.

\section{9 - Referências Bibliográficas}

Atutor. Atutor: Learning Content Management System. 2005. Disponível em: $<$ http://www.atutor.ca/>. Acesso em: 15 ago 2005.

Bicudo, S. F. Uma Abordagem Integrada para Educação A Distância Usando Gestão de Processos e Simulação de Sistemas. Tese de Doutorado do Curso de PósGraduação em Computação Aplicada CAP/INPE. Instituto Nacional de Pesquisas Espaciais - INPE. São José dos Campos, 2006.

Blackboard Inc. Blackboard Oficial Site. Disponível em: $<$ http://www.blackboard.com/>. Acesso em: 15 ago 2005.

Caci Products Company. Simprocess User's Manual - V. 4.0. 2004.

Claroline.Net. Claroline. Open Source e-Learning. 2005. Disponível em: $<$ http://www.claroline.net/>. Acesso em: 15 ago 2005.

Helic D., Hrastnik J., Maurer H. An Analysis of Application of Business Process Management Technology in E-Learning Systems, In Proceedings of E-Learn 2005, pages 2937-2942, AACE, Charlottesville, USA, 2005.

EzComponent. Disponível em <http://ezcomponents.org/>. Acesso: 13 de abril de 2008.

Greenberg, L. LMS and LCMS: What's the Difference? Disponível em: <http://www.learningcircuits.org/2002/dec2002/greenberg.html>. Acesso: 03 mar 2005.

Kienbaum, G.S.; Bicudo, S.F.; Marcondes, V. P. P. Construção e Gestão de Conteúdo Educacional Usando Gestão de Processos e Simulação de Sistemas. In: 
CICLO DE PALESTRAS SOBRE NOVAS TECNOLOGIAS NA EDUCAÇÃO, 2006, Porto Alegre. Anais do VII Ciclo de Palestras sobre Novas Tecnologias na Educação. Porto Alegre, CINTED - UFRGS, 2006. V.1. pp 1-11.

Lin J.; Ho C.; Sadiq, W.; Orlowska, M. O.. Using Workflow Technology to Manage Flexible e-Learning Services. Journal of Educational Technology \& Society, Volume 5, Issue 4, 2002. IEEE Learning Technology Task Force, October 2002.

Núcleo de Informática Aplicada à Educação (NIED) Universidade Estadual de Campinas (UNICAMP). Disponível em: http://teleduc.nied.unicamp.br/teleduc/. Acesso em: 11 set 2005

Marcondes, V. P. P. ;Kienbaum, G.S.; Bicudo, S.F. Aplicação da Tecnologia de Workflow na Modelagem e Gestão do Processo em Educação a Distância. In: VIII ENCONTRO VIRTUAL EDUCA , 2007, São José dos Campos. VIII Encontro Virtual Educa, 2007.

Moodle 2004. Disponível em: <http://www.moodle.org/>. Acesso em: 15 ago 2005.

Webct Inc. WebCT: Learning Without Limits. Disponível em: <http://www.webct.com/>. Acesso em: 15 ago 2005. 